\title{
Effects of Brief Fitbit Use on Physical Activity \& Cardiometabolic Risk
}

\author{
Melanie M. Adams ${ }^{1, *}$, Chelsea Freleng ${ }^{2}$ \\ ${ }^{1}$ Department of Human Performance \& Movement Sciences, Keene State College, Keene, NH, USA \\ ${ }^{2}$ Peterson Physical Therapy, Amagansett, NY, USA \\ *Corresponding author: madams4@keene.edu
}

Received August 01, 2018; Revised September 12, 2018; Accepted September 28, 2018

\begin{abstract}
Wrist-worn fitness trackers are popular consumer products, but few studies have documented their use as a stand-alone intervention tool. This study examined the effect of wearing the Fitbit Flex on moderate to vigorous physical activity (MVPA), sedentary behavior (SB), self-efficacy (SE), and cardiometabolic risk factors. Participants ( $n=28, m$ age $=50.5$ yrs) wore the Fitbit for 3 weeks, then were randomly assigned to continue for 3 more weeks (6week group) or discontinue use (3-week group). Participants were not physically active prior to the study. Accelerometers recorded weekly, work-week and weekend MVPA and SB at baseline and post. BMI, waist circumference, and blood pressure were measured baseline and post. A self-efficacy questionnaire was administered baseline, mid-point, and post. MVPA significantly increased in both groups. The 6-week group performed better on weekend MVPA. SB did not change. Waist circumference decreased significantly in both groups. Overall, there was no change in SE. However, the hypothesized pattern between groups was found. The 6-week group had a small increase in SE while the 3-week group declined non-significantly. Fitbit fitness trackers are effective at increasing MVPA and impact waist circumference, in as little as 6 weeks. However, the mechanism for behavior change is unclear and needs further study. This research is challenging as the features of fitness trackers are constantly changing.
\end{abstract}

Keywords: Fitbit, physical activity, sedentary behavior, cardiovascular disease, diabetes

Cite This Article: Melanie M. Adams, and Chelsea Freleng, "Effects of Brief Fitbit Use on Physical Activity \& Cardiometabolic Risk." Journal of Physical Activity Research, vol. 3, no. 2 (2018): 82-88. doi: 10.12691/jpar-3-2-4.

\section{Introduction}

Fitness trackers are popular tools for monitoring physical activity. These wrist-worn accelerometers estimate steps, minutes and intensities of physical activity (light, moderate or vigorous). More expensive models also measure heart rate and distance. Fitbit Inc. has manufactured fitness trackers since 2007. The company reported 25.4 million active users at the end of 2017 [1]. Fitbit's relatively low cost $(\$ 100.00-150.00)$, compared to research accelerometers [Actigraph, activ PAL] [2] make them attractive for community and workplace health promotion programs. Increased physical activity has been seen in college students [3], women at risk for breast cancer [4], postmenopausal women [5] and sedentary hospital employees [6] when Fitbits were part or all of a long-term intervention (16-24 weeks).

Multiple behavior change strategies and theories are incorporated into fitness trackers including; goal setting, self-monitoring, immediate and delayed feedback, behavioral cues, mastery experience, social support, social comparison and rewards [7,8]. In 2015, the Fitbit Flex displayed a series of dots to show progress towards the daily step goal. Five dots signified completion of the goal. The device also vibrated when the step goal was achieved. An online application called the Fitbit Dashboard (Figure 1) comes with the tracker and provided more data in a colorful display in real time. The dashboard allowed users to view daily progress and their physical activity over previous days, weeks, and months. Badges for personal bests or accumulated distances (miles across state) are displayed.

These features appear to highlight success and develop a sense of mastery, thus improving self-efficacy for physical activity. Lyons and colleagues [7] gave the Fitbit strong marks for being theoretically-based. Though, which theory or feature is responsible for an increase in physical activity with fitness tracker use is not known.

Two studies have measured self-efficacy during the use of fitness trackers. Chen et al. [9] placed the Fitbit Flex on adolescents and O'Brien and colleagues [10] used the Nike Fuel Band in older female adults. There was a significant increase in self-efficacy over 6 months in the Chen et al. study along with improved frequency of physical activity. However, the Fitbit was only part of the intervention, which included an online educational session and motivational text messages [9]. Self-efficacy did not change in the older women when the tracker was the only intervention over 12 weeks [10]. 


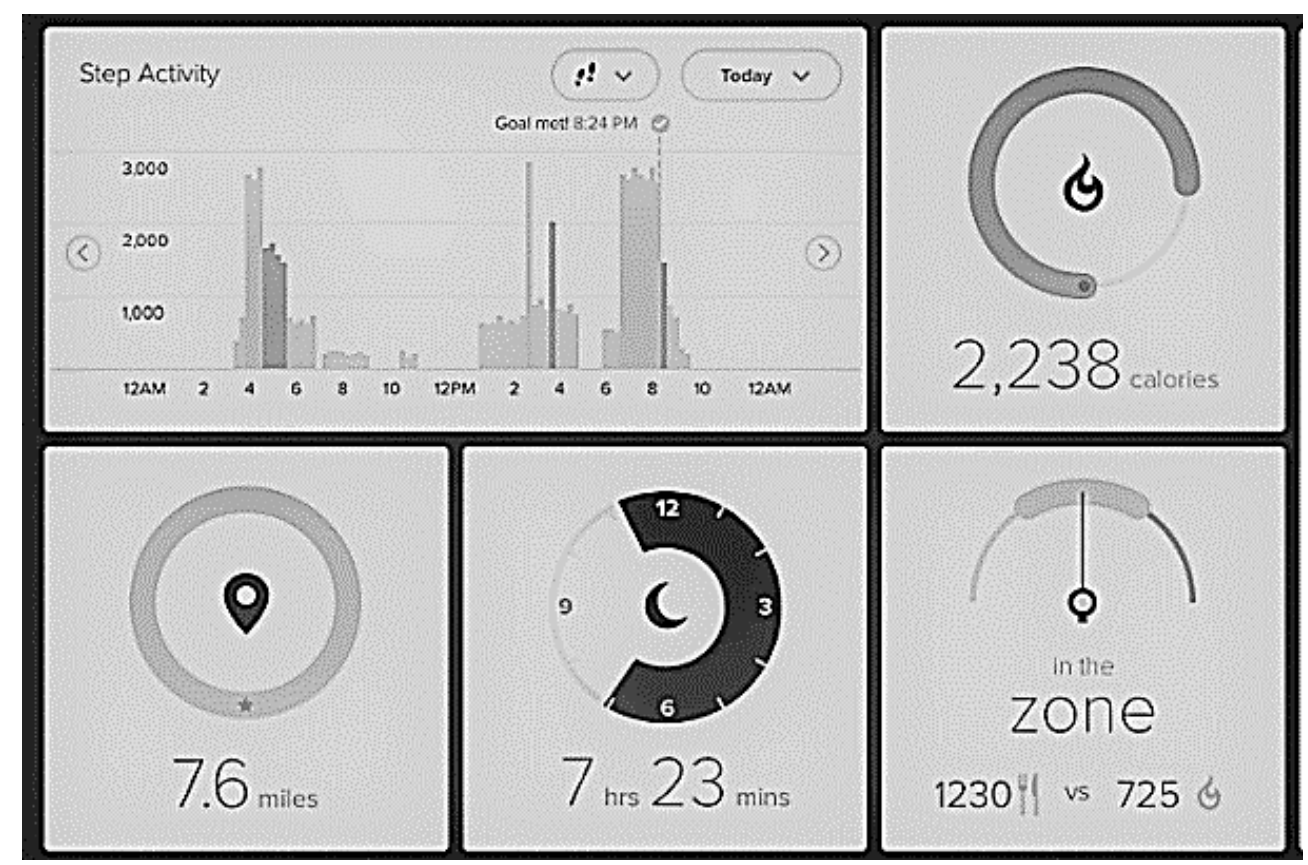

Figure 1. The Fitbit Dashoard

Clearly, more research is needed to determine if fitness trackers improve self-efficacy. Additionally, interventions less than 12 weeks have not been tested. The purpose of this study was to examine the effect of short-term use the Fitbit Flex on physical activity, sedentary behavior, self-efficacy for exercise, blood pressure, and waist circumference in adults who do not exercise. This study further considered what effect discontinuing Fitbit use after 3 weeks has on physical activity, sedentary behavior and self-efficacy.

\section{Materials and Methods}

\subsection{Participants}

After IRB approval, employees of a small college were recruited through a campus-wide email. Inclusion was limited to self-identified non-exercisers, ages 25-65, who had no medical restrictions for physical activity, were not using a standing desk nor whose job required physical activity such as grounds keeping or maintenance. As an incentive, a free Fitbit Flex was given to participants.

Thirty participants were enrolled; 28 completed all measures. The sample was predominantly female $(n=20)$, middle-aged ( $m 50.5$ yrs) and white (100\%). Mean BMI was 29.32 ( $s d$ 6.01) and 50\% had elevated blood pressure at baseline. The sample was insufficiently active. The average accelerometer-determined accumulated weekly physical activity was 65.3 minutes ( $s d$ 32.79). Only one participant met the 150 minute guideline. After randomization, post-hoc analysis found no significant differences in age, blood pressure category, or gender between the groups. BMI was significantly higher (26.99 to 31.52) in the 3-week group than the 6-week group. The 6-week group also had significantly higher baseline physical activity minutes $(p=0.03)$.

\subsection{Intervention}

The Fitbit Flex fitness tracker and Fitbit Dashboard were the only intervention tools used in this study. After a researcher installed the Fitbit software to their computer and/or cell phone, participants received a 10-minute tutorial on wearing the tracker and using the Dashboard. This included wearing and charging the device, logging-in, setting up their profile, goals and notifications, and viewing their data. Participants were free to use the Fitbit as any consumer would. They could engage with the online information as often as they liked or not at all. The default goals for daily steps $(10,000)$, weekly steps $(70,000)$, daily physical activity (30 minutes), and daily distance (5 miles) were used unless the participant indicated different goals.

All participants were instructed to wear the Fitbit for the next 3 weeks. They received one text message reminder each morning for the first 3 days of the intervention. In order to assess any carryover effects, half of the participants were randomized to discontinue wearing the Fitbit at the beginning of week 4 . Participant-identification codes were entered into a random number generator to create 2 groups for comparison; 6-week $(n=15)$ and 3-week $(n=13)$.

\subsection{Measures}

Study measures and procedures were explained to participants prior to completing the IRB-approved informed consent document. There were 2 assessment points; baseline and post for MVPA, SB, and the cardiometabolic risk factors (BMI, waist circumference, blood pressure). Self-efficacy was assessed 3 times; baseline, mid-point, and post.

\subsubsection{Physical Activity \& Sedentary Behavior}

The Actigraph GXT-3 accelerometer was worn by each participant to objectively measure minutes of MVPA (work-week, weekend and weekly) and minutes of SB 
(work-week, weekend and weekly) the week prior to the intervention and again in the final week. The device was worn on an elastic belt over the left hip, slightly medial to the anterior superior iliac crest. The accelerometer recorded counts in 60 second epochs at a frequency of $60 \mathrm{~Hz}$. The Troiano [11] cut points were applied to establish the intensities of physical activity. The Actigraph inclinometer differentiated between sitting and standing and tallied minutes of SB. Continuous non-movement for 60 minutes was labeled as non-wear time and removed from the analysis. Accelerometer data was further segmented into work-week (Monday-Friday), weekend (Saturday-Sunday) periods. The first 3 weeks of Fitbit steps were reviewed to determine how many participants reached 10,000 steps.

\subsubsection{Cardiometabolic Risk Factors}

Body Mass Index (BMI), waist circumference (WC), and blood pressure were assessed. Height and weight were measured using standard procedures on a physician's balance scale and stadiometer. The data was transformed into BMI using the formula: $\left(\frac{l b s}{\text { inches xinches }}\right) \times 703$. Mid-abdominal WC was taken using a Gulick tape measure. Tension was applied immediately after exhale and the average of two attempts was recorded in centimeters. A disease risk category was assigned to each participant at baseline and post using the NIH combined scale for BMI and waist circumference [12]. The terms were no risk, increased, high, very high or extremely high.

A standard sphygmomanometer was applied to the nondominant upper arm and inflated to $220 \mathrm{mmHg}$. A trained researcher determined the systolic and diastolic pressures using a stethoscope over the brachial artery. Participants were categorized as normal, elevated (systolic $=120-129 \mathrm{mmHg}$ and diastolic $<80 \mathrm{mmHg}$ ), or hypertensive (systolic $\geq 130 \mathrm{mmHg}$ or diastolic $\geq 80 \mathrm{mmHg}$ ) based on the 2017 ACC/AHA guidelines [13].

\subsubsection{Self-efficacy for Exercise}

Self-efficacy was measured using the Exercise Confidence Survey developed by Sallis and colleagues [14]. This 10-item questionnaire rates confidence to engage in exercise under different circumstances; i.e. after a long day or when other tasks are required. Participants responded using a 1-5 Likert-scale. Labels for 1, 3, and 5 are "I know I cannot", "maybe I can", and "I know I can". The mean score was calculated for each survey. Scores greater than 3 indict moderate to high levels of self-efficacy for exercise.

\subsection{Statistical Analysis}

Descriptive statistics were computed for all measures at all time-points. Pearson correlations examined relationships between the MVPA and SB variables and SE. Four separate repeated measures analysis of variance (RMANOVA) were run to determine changes in MVPA (weekly, work-week and weekend), SB (weekly, work-week and weekend), cardiometabolic risk factors (BMI, waist circumference, blood pressure), and self-efficacy. The RMANOVA used within and between subjects designs in order to assess differences between the 6-week and 3 -week groups. Follow up t-tests were conducted and effect sizes (Cohen's $d$ ) were calculated for any significant result. Cohen's $d$ coefficients were categorized as small $(\geq 0.20)$, moderate $(0.50-.79)$ or large $(\geq 0.80)$.

$\mathrm{A} \mathrm{chi}{ }^{2}$ test of independence determined if there was a relationship between group and a change in blood pressure or disease risk. Additionally, frequency counts examined how many participants met the 10,000 Fitbit step goal and 150 minutes of MVPA a week. P-value was set at $\leq 0.05$ for all statistical tests.

\section{Results}

\subsection{Physical Activity}

Both groups significantly increased all MVPA (weekly, work-week and weekend). Weekly MVPA increased from 65.3 ( $s d$ 32.8) to 98.6 ( $s d$ 44.8) minutes (Figure 2). Work-week minutes went from 32.4 ( $s d$ 17.5) to 42.3 ( $s d 19$ ).

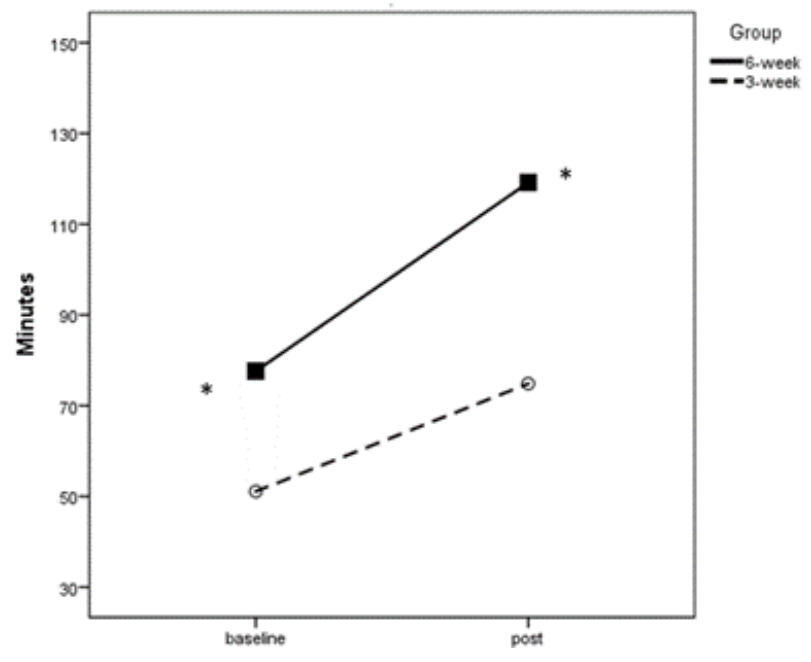

Figure 2. Weekly MVPA by Group

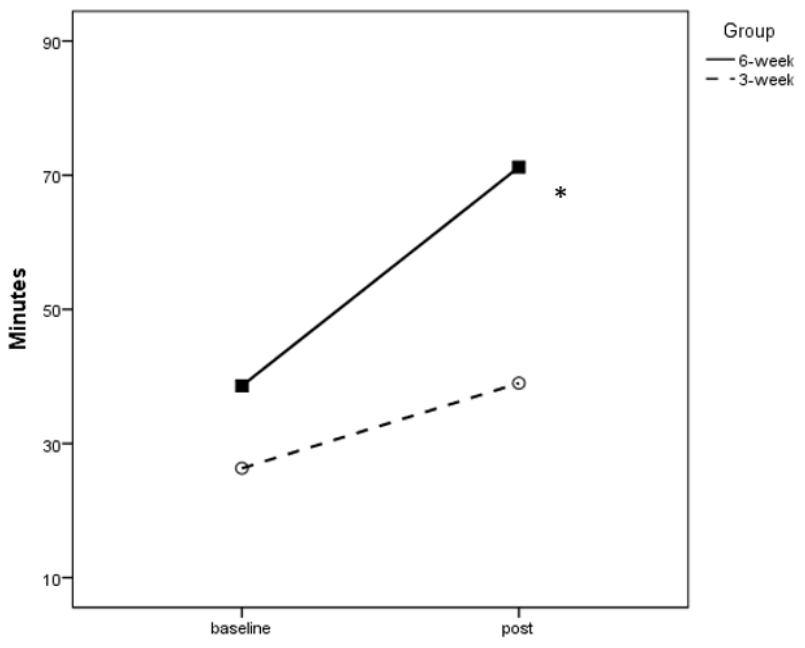

Figure 3. Weekend MVPA by Group

Weekend physical activity had the largest change from 32.9 ( $s d 20.4$ ) to 52.3 ( $s d$ 30.9) minutes (Figure 3). Strong effect sizes were found for weekly MVPA $(d=.86)$ and weekend MVPA $(d=.91)$. Significant differences exist between the groups at baseline $(p=0.03)$ and post $(\mathrm{p}=0.01)$ for weekly MVPA. At post, groups differed in weekend 
( $p=0.00)$ MVPA. The 6-week group was higher in all cases. Effects in the 3-week group were moderately strong ( $d=.60-.72)$, while the effects in the 6-week group were larger $(d=1.117-1.37)$. No significant group $\mathrm{x}$ time interactions were found for weekly or work-week MVPA. A trend towards significance was seen for weekend MVPA $(p=0.07)$.

Though minutes of MVPA were substantially improved, only 5 participants met the 150 minutes guideline at post. All were in the 6-week group. Similarly, only 8 participants met the 10,000 Fitbit step goal at least once in the first 3 weeks. Six of these participants were in the 6-week wear group.

\subsection{Sedentary Behavior}

Overall, there was no significant change in weekly, work-week, or weekend minutes of SB. Total weekly sitting time increased non-significantly from 839.6 to 887 minutes. This was mostly due to greater in work-week SB in the 6-week group (472 to 538 minutes). The 3-week group decreased work-week SB non-significantly (481.6 to 452.1 minutes). Group differences and group $\mathrm{x}$ time interaction neared significance $(p=0.06)$. Follow up t-tests found no significant changes.

\subsection{Cardiometabolic Risk Factors}

Waist circumference was significantly improved from baseline to post $(p=0.00)$ in all participants, without a group $\mathrm{x}$ time interaction. Paired t-tests revealed that waist circumference decreased significantly in the 6-week group $(p=0.05)$ and the 3 -week group $(p=0.04)$. The effect size in both groups was small $(d=0.24-.28)$. There was a trend $(p=0.07)$ for group differences; with the 3-week group being non-significantly higher than the 6-week group at both time points $(p=0.06,0.07)$. There was no change in BMI during the study. The groups were significantly different at baseline $(p=0.04)$ and post $(p=0.05)$; without a group $\mathrm{x}$ time interaction. Four participants (2 male, 2 female) decreased their NIH disease risk level. However, 2 (female) increased their risk categories. There were no significant changes to systolic or diastolic pressures. However, the number of participants with elevated blood pressure decreased from 14 to 11 . $\mathrm{Chi}^{2}$ analysis revealed no differences between groups on blood pressure category or NIH disease risk. Table 1 contains the group means, $\mathrm{p}$ values, and effect sizes for the risk factors.

Table 1. Means, sd, effect size $\&$ p-values for Risk Factors

\begin{tabular}{|c|c|c|c|c|}
\hline \multirow{5}{*}{ 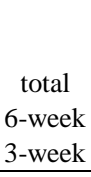 } & \multicolumn{4}{|c|}{ Waist Circumference } \\
\hline & baseline & post & $d$ & $p$ \\
\hline & $101.96(14.75)$ & $98.34(15.31)$ & 0.24 & $0.00^{\#+\$}$ \\
\hline & $97.11(12.26)$ & $93.73(12.39)$ & 0.28 & $0.05^{*}$ \\
\hline & $107.55(15.83)$ & $103.67(17.04)$ & 0.24 & $0.03 *$ \\
\hline \multirow{5}{*}{$\begin{array}{c}\text { total } \\
\text { 6-week } \\
\text { 3-week }\end{array}$} & \multicolumn{4}{|c|}{ BMI } \\
\hline & baseline & post & $d$ & $p$ \\
\hline & $29.09(6.0)$ & $29.17(6.28)$ & -0.01 & $0.70^{\$}$ \\
\hline & $27.0(3.62)$ & $27.0(3.79)$ & 0.00 & \\
\hline & $31.52(7.31)$ & $31.67(7.7)$ & -0.15 & \\
\hline \multirow{5}{*}{$\begin{array}{c}\text { total } \\
\text { 6-week } \\
\text { 3-week }\end{array}$} & \multicolumn{4}{|c|}{ Systolic / Diastolic Blood Pressure } \\
\hline & baseline & post & $d$ & $p$ \\
\hline & $126(17) / 80(9)$ & $122(11) / 78(7)$ & $0.29 / .26$ & 0.137 \\
\hline & $124(17) / 78(9)$ & $119(12) / 76(7)$ & $0.36 / .26$ & \\
\hline & $127(17) / 81(10)$ & $124(9) / 80(6)$ & $0.23 / .13$ & \\
\hline
\end{tabular}

${ }^{\#}$ within subjects $\mathrm{p} \leq 0.05$, "between subjects trend $\mathrm{p} \leq 0.07$, ${ }^{\text {sig group }}$ differences: 3 -week $>6$-week, , sig change from baseline.

\subsection{Self-efficacy}

There was no significant change in SE or interaction between groups according to the RMANOVA. The group $\mathrm{x}$ time graph (Figure 4) pointed towards the hypothesized result so follow up t-tests were used. The 6-week group had a significant increase from baseline to post $(p=0.04)$, with a moderate effect size $(d=0.63)$, while no change was found in the 3-week group.

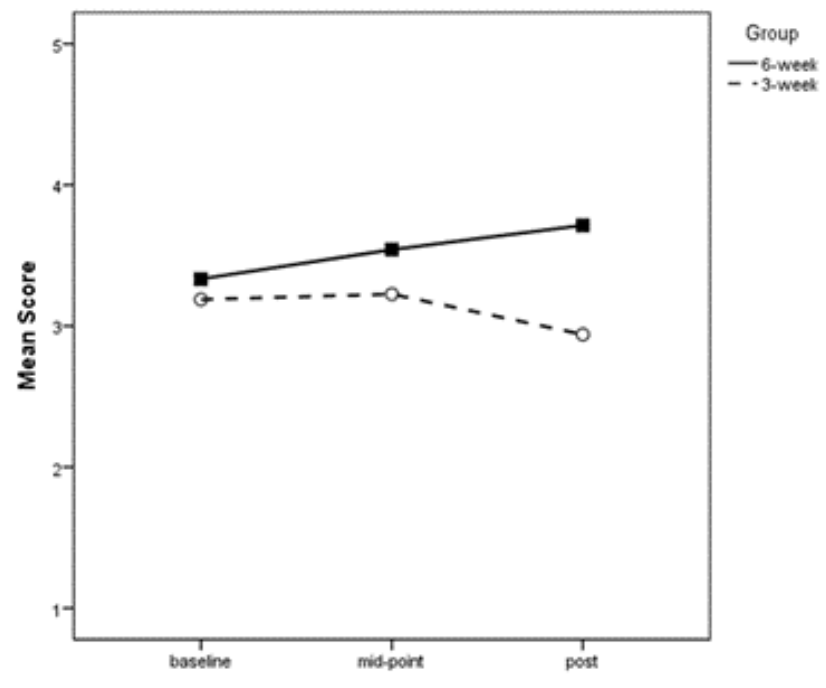

Figure 4. Self-efficacy by Group

An independent t-test revealed a trend for group differences at the post measure $(p=0.06)$. Pearson correlations found that mid-point SE was moderately related to post weekly $(r=.34, p=0.04)$ and post weekend $(\mathrm{r}=.38, \mathrm{p}=0.05)$ MVPA. Interestingly, post SE was not related to MVPA.

\section{Discussion}

This brief Fitbit intervention was effective at increasing physical activity in non-exercising adults. The mean increase for all participants was 33 minutes of weekly MVPA. Surprisingly, the 3-week group had an increase 23 minutes at the post assessment, even after 3 weeks without the tracker. This, points to some lasting influence. It is important to put these finding in context of the guidelines for health-producing physical activity. Only 5 of the 28 participants reached 150 minutes of MVPA. All of these were in the 6-week group. The MVPA results are similar to the workplace study by Losina et al. [6] where the average improvement was 31 minutes. Those with better compliance with the Fitbit had substantially higher increases in physical activity. Here as well, greater exposure to the Fitbit produced better outcomes as the 6-week group had more minutes per week, were more likely to meet the step goal and the physical activity guideline than the 3-week group. Our results are not nearly as robust as CadamusBertram et al. [5] who reported a weekly increase of 62 minutes after 16 weeks. Hartman et al. [4] saw a daily increase in MVPA of 15 minutes for participants that used Fitbit in conjunction with counseling at 6 months. Possibly, the shorter intervention period explains the difference in volume between this study and the earlier studies. 
Unlike prior research, work-week and weekend physical activity were analyzed separately in this study. All time periods showed a significant increase from baseline. The greatest effect was seen in weekend MVPA, especially in the 6-week group. While this study was initiated as a workplace intervention, participants appear to be better able to add physical activity to their non-work days than to their work-week. The physical activity guideline breaks the weekly 150 minutes into a frequency of at least 5 days a week [15]. This requires activity on at least 3 work days. Health and fitness professionals may need to help reduce work-related barriers so that the frequency recommendation can be achieved. Future workplace interventions should document which days of the week participants are physically active. Greater frequency should be sought for cardiometabolic benefits [16].

Sedentary behavior was unchanged in both groups. This is another workplace specific variable that is important to cardiometabolic health. Frequent breaks from sitting are associated with lower waist circumference, cholesterol and glucose [17]. Increases in light physical activity are necessary to reduce sedentary time. Our participants understood the need to increase daily steps and movement but interpreted that to mean MVPA, though no specific instructions were given. The original Fitbit provided information on sedentary time but that feature was removed in the Flex. Without specific feedback on sitting time or breaks, it is understandable why participants did not change this behavior. Newer Fitbits track steps per hour and designate periods of less than 250 steps per hour as sitting. Users can set an alarm cue for them to move at least once an hour. Some workplace barriers exist when discussing reducing sedentary behavior such as perception of lack of productivity by supervisors or co-workers, difficulty in changing something so habitual, and the awkwardness of standing when others are sitting [18]. A meta-analysis of interventions to reduce occupational sitting suggests using workstations that allow employees to stand, walk or bike as alternatives to taking breaks [19]. Unfortunately, fitness trackers worn at the wrist will not pick up much of this movement as hands will be on the workstation and not free moving.

With the increased physical activity came a significant decrease in waist circumference but no change in BMI. The average reduction in waist circumference was $3.6 \mathrm{~cm}$. This time, the 3-week group out performed with a loss of $3.88 \mathrm{~cm}$, though they started at a significantly higher mark. The effect was small for both groups. Waist circumference is an important risk factor for Type 2 Diabetes. This study provides further evidence that improvements can be made without weight loss through moderate increases in physical activity [20]. O'Brien and colleagues [10] also documented a significant decrease in waist circumference during a fitness tracker intervention with older adults over 12 weeks. In the current study, 4 participants ( 2 male, 2 female) decreased their disease risk. This clinically important change to cardiometabolic risk occurred relatively quickly and with a cost of only $\$ 100.00$ per participant.

The underlying mechanism for the increase in physical activity remains unclear. Self-efficacy did not change significantly though there was a small increase in the 6-week group. Figure 4 is promising. While not significant, SE decreased when the Fitbit was removed. Mid-point SE positively correlates to post MVPA, so the small improvement in the 6-week also contributed to greater MVPA A key component of building self-efficacy is mastery experience. When a Fitbit user meets their preset goal, the feedback should enhance a sense of accomplishment. Interestingly, only 8 participants achieved the 10,000 step mark during the first 3 weeks of the study. Since few experienced this success, it stands to reason that self-efficacy would not increase substantially by the midpoint. Unfortunately, we could not track goal completion after the $3^{\text {rd }}$ week due to our limited number of Fitbits. At the randomization point, we provided the 6-week participants with their own Fitbit and no longer had access to the dashboards. Goal achievement should be considered in any future research linking SE and fitness trackers. Also, a larger sample would possibly improve the self-efficacy finding. O'Brien et al [10], found no change in self-efficacy with a similar sized sample. Chen and colleagues [9] saw improvements to self-efficacy with 40 participants, but augmented the Fitbit intervention with additional tools.

There are multiple behavior change techniques incorporated into the Fitbit. Behavioral cueing, goal setting, selfmonitoring, or the reward of having the dashboard light up may be contributing to physical activity more than selfefficacy. The psychosocial-behavioral elements of fitness trackers need further study to determine which are most impactful. This is especially true as features change frequently with advancing technology.

In this study, we took the Fitbit away from half the participants after 3 weeks to simulate what a loss of interest in the device would do to physical activity and self-efficacy. There was some carry over effect as those participants did have greater MVPA at post. Future studies should take a longitudinal approach and look for relationships between personal characteristics (self-efficacy, stage of change, history and type of physical activity), length of tracker use and health outcomes. This could lead to a better understanding of when and for whom fitness trackers are effective.

With any real-world intervention, there are a number of limitations. First, this sample is small and homogenous. Participants had high social-economic status and no history of diabetes, stroke or heart disease. The intent was to target a sedentary population and therefore employees that worked in maintenance, delivery, and landscaping were excluded. Statistical power was sufficient for continuous data such as minutes per week or centimeters. However, self-efficacy scales have fewer integers making it harder to find statistical significance without a large sample. Next, weather may have played a role. Participants were grouped into 3 cohorts based on their preferred start; late-January, mid-February or mid-March. Post-hoc analysis found an interaction for work-week MVPA by cohort; with the last cohort having the greatest improvement. We did not investigate the intentions or goals our participants had for enrolling in the study. We missed an opportunity to link stage of change, goal setting, or outcome expectations to behavior change. As mentioned above, more careful study is needed to determine where in the behavior change progress do trackers provide the most benefit. 
Another limitation of fitness tracker research is the inability to keep up with the advancing technology. There are important differences between the Fitbit discussed here and models currently available. Newer Fitbits have a digital output that gives an exact read out of the number of steps, minutes of physical activity, heart rate, calorie expenditure, and distances travelled on the wristband. This is significantly more advanced from the Flex model used in this study, which had a 5 dot system for seeing progress for steps. These improvements in immediate feedback may better outcomes or may be overwhelming to the user. Testing every new model is not feasible, but a core set of features should be evaluated to determine which provide the most support for increasing physical activity.

\section{Conclusion}

Fitness trackers, like the Fitbit do improve physical activity behavior, both short-term and over serval months. What is still unknown are which specific features of the tracker are initiating the behavior change, which are maintaining it and who is most likely to benefit from using it. As these devices have proven to be moderately impactful, they should be included in community and workplace health promotion programming when possible.

Our participants were not given any guidance in their behavior change. We were specifically trying to test the effect of using the Fitbit as a regular consumer, rather than as part of a health promotion program. To improve outcomes more behavior change coaching is needed. Specifically, goals should be self-determined rather than the default 10,000 steps or 30 minutes of physical activity a day. Experiencing success is key. Goal setting activities can help establish challenging yet achievable targets. Participants should develop 2-3 action steps for meeting their goal. For workplace interventions, planning and problem solving strategies to make physical activity more accessible during the work-week are needed.

\section{Acknowledgements}

The authors would like to thank Healthy KSC and the Summer Undergraduate Research Fund for their generous support of this project.

\section{Competing Interests}

The authors have no competing interests.

\section{Abbreviations}

BMI - Body Mass Index

$d$-Cohen's d

$\mathrm{NIH}$ - National Institutes of Health

MVPA - Moderate to Vigorous Physical Activity

RMANOVA - Repeated Measures Analysis of Variance

SE - Self-efficacy

$s d-$ Standard Deviation
SB - Sedentary Behavior

WC - Waist Circumference

\section{References}

[1] Fitbit, Inc., "Fitbit Reports \$571M Q4'17 and \$1.616B FY'17 Revenue," Business Wire, 2018, Available: https://www.businesswire.com/news/home/20180226006448/en/.

[2] Sylvia, L.G., Bernstein, E.E., Hubbard, J.L., Keating, L., and Anderson, E.J., "A Practical Guide to Measuring Physical Activity," Journal of the Academy of Nutrition and Dietetics, 114 (2), 199-208, 2014.

[3] Rote, A.E., "Physical activity intervention using Fitbits in an introductory college health course," Health Education Journal, 2016.

[4] Hartman, S.J., Nelson, S.H., Cadmus-Bertram, L.A., Patterson, R.E., Parker, B.A., and Pierce, J.P., "Technology- and PhoneBased Weight Loss Intervention: Pilot RCT in Women at Elevated Breast Cancer Risk," American Journal of Preventive Medicine, 51 (5), 714-721, 2016.

[5] Cadmus-Bertram, L.A., Marcus, B.H., Patterson, R.E., Parker, B.A., and Morey, B.L., "Randomized Trial of a Fitbit-Based Physical Activity Intervention for Women," American Journal of Preventive Medicine, 49 (3), 414-418, 2015.

[6] Losina, E., Smith, S., Usiskin, I., Klara, K., Michl, G., Deschande, B., and al., e., "Implementation of a workplace intervention using financial rewards to promote adherence to physical activity guidelines: a feasibility study," BMC Public Health, 17 (1), 2017.

[7] Lyons, E.J., Lewis, Z.H., Mayrsohn, B.G., and Rowland, J.L., "Behavior Change Techniques Implemented in Electronic Lifestyle Activity Monitors: A Systematic Content Analysis," Journal of Medical Internet Research, 16 (8), 2014.

[8] Mercer, K., Li, M., Giangregorio, L., Burns, C., and Grindrod, K., "Behavior Change Techniques Present in Wearable Activity Trackers: A Critical Analysis," JMIR Mhealth and Uhealth, 4, 2016,

[9] Chen, J.L., Guedes, C.M., Cooper, B.A., and Lung, A.E., "ShortTerm Efficacy of an Innovative Mobile Phone Technology-Based Intervention for Weight Management for Overweight and Obese Adolescents: Pilot Study," Interactive Journal of Medical Research, 6 (2), 2017.

[10] O'Brien, T., Troutman-Jordon, M., Hathaway, D., Armstrong, S., and Moore, M., "Acceptability of wristband activity trackers among community dwelling older adults.," Geriatric Nursing, 36 (2), S21-25, 2015 .

[11] Troiano, R., "Translating accelerometer counts into energy expenditure: advancing the quest," Journal of Applied Physiology, 100 (4), 1107-1108, 2006

[12] NIH, "Classification of Overweight and Obesity by BMI, Waist Circumference, and Associated Disease Risks," 2018, Available: https://www.ncbi.nlm.nih.gov/pubmed/.

[13] Whelton, P.K. et al., "Guideline for the Prevention, Detection, Evaluation, and Management of High Blood Pressure in Adults: A Report of the American College of Cardiology/American Heart Association Task Force on Clinical Practice Guidelines," Journal of the American College of Cardiology, 71 (19), e127-248, 2017.

[14] Sallis, J.F., Pinski, R.B., Grossman, R.M., Patterson, T.L., and Nader, P.R., "The development of self-efficacy scales for healthrelated diet and exercise behaviors," Health Education Research, 3 (3), 283-292, 2017.

[15] DHHS, "Physical Activity Guidelines for Americans," 2008 , Available: https://www.hhs.gov/fitness/be-active/physical-activityguidelines-for-americans/index.html.

[16] Garber, C. et al., "Quantity and Quality of Exercise for Developing and Maintaining Cardiorespiratory, Musculoskeletal and Neuromuscular Fitness in Apparently Healthy Adults" Medicine and Science in Sports and Exercise, 43 (7), 1334-1359, 2011,

[17] Healy, Dunstan, Salmon, J., Cerin, E., Shaw, J.E., Zimmet, P.Z., and Owen, N., "Breaks in sedentary time: beneficial associations with metabolic risk," Diabetes Care, 31 (4), 661-666, 2008.

[18] De Cocker, K., Veldeman, C., De Braeckman, L., Owen, N., Cardon, G., and De Bourdeaudhuij, I., "Acceptability and 
feasibility of potential intervention strategies for influencing sedentary time at work: focus group interviews in executives and employees," International Journal of Behavioral Nutrition and Physical Activity 12 (22), 2015.

[19] Neuhaus, M. et al., "Reducing occupational sedentary time: a systematic review and meta-analysis of evidence on activity- permissive workstations," Obesity Reviews, 15 (10), 822-838, 2018.

[20] Camhi, S.M., Sisson, S.B., Johnson, W.D., Katzmarzyk, P.T., and Tudor-Locke, C., "Accelerometer-determined moderate intensity lifestyle activity and cardiometabolic health," Preventive Medicine, 52 (5), 358-360, 2011. 